\title{
Lattice Boltzmann Study of a Vortex Ring Impacting Spheroidal Particles
}

\author{
Chunlong Yu, Haibo Huang* and Xiyun Lu \\ Department of Modern Mechanics, University of Science and Technology of China, \\ Hefei 230027, China \\ Received 4 November 2013; Accepted (in revised version) 21 February 2014 \\ Available online 28 May 2014
}

\begin{abstract}
Interaction of vortex rings with solid is an important research topic of hydrodynamic. In this study, a multiple-relaxation time (MRT) lattice Boltzmann method (LBM) is used to investigate the flow of a vortex ring impacting spheroidal particles. The MRT-LBM is validated through the cases of vortex ring impacting a flat wall. The vortex evolution due to particle size, the aspect ratio of a prolate particle, as well as Reynolds $(R e)$ number are discussed in detail. When the vortex ring impacting a stationary sphere, the primary and secondary vortex rings wrap around each other, which is different from the situation of the vortex ring impacting a plate. For the vortex ring impacting with a prolate spheroid, the secondary vortex ring stretches mainly along the long axis of the ellipsoid particle. However, it is found that after the vortex wrapping stage, the primary vortex recovers along the short axis of the particle faster than that in the long axis, i.e., the primary vortex ring stretches mainly along the short axis of the particle. That has never been address in the literature.
\end{abstract}

AMS subject classifications: 76D17

Key words: Vortex interaction, lattice Boltzmann method, spheroid, particle.

\section{Introduction}

The study of vortex ring interactions with solid bodys has attracted much attention. The possible reasons are that the experiments are easy to carry out and the results would give physical insights to more complicated flow fields dominated by vorticities.

The simplest interaction between a vortex and a solid body may be a vortex ring impacting on a flat wall. This case is helpful to understand the topological changes in evolving vortex structures [1]. This topic has been studied extensively through experiment and numerical simulations [1-5]. For example, Chu [5] studied the vortex structure

*Corresponding author.

Email: huanghb@ustc.edu.cn (H. B. Huang) 
and the associated surface force with $500 \leq R e \leq 2000$ numerically and experimentally. In the literature, the following procedure is identified $[1,5]$. When the vortex ring approaches the wall, a boundary layer with vortices of opposite signs will form, which is a viscous response in the vicinity of the wall $[6,7]$ due to the non-slip wall boundary condition. In the procedure, the toroidal radius of the vortex ring would expand. Due to vortex stretching, the vortex core shrinks but its vorticity is intensified [1]. Finally, a secondary vortex and even a tertiary vortex ring may be generated from the boundary layer and leave the boundary.

There are also some numerical studies on the interaction of the ring with an inclined wall. Liu [8] used the vortex particle-in-cell method and a hybrid Eulerian-Lagrangian method to simulate the unsteady shear flow induced by a vortex ring impacting a flat wall with angle $38.5^{\circ}$. Liu [8] proved that the helical winding of vortex lines behaved more prominent and the secondary vortex core is thicker at a high $R e$. Cheng et al. [1] studied a vortex ring impacting a flat wall with an angle of $0 \leq \theta \leq 40$ and $100 \leq R e \leq 1000$. He observed the development of the primary vortex ring and the pattern of the secondary vorticity generation, and made a investigation on the effect of $\theta$ and $R e$.

On the other hand, vortex interaction with a stationary and rotating spheroidal particle, which probably represents the simplest interaction of a vortex with a body, receives less attention $[9,10]$. To the best of our knowledge, the following two works were carried out in the literature. One is the experimental study of a vortex ring impacting a sphere [9]. In the experiment, a neutrally buoyant sphere was free to move in response to the impulse delivered by a vortex ring in water. The other work is a numerical study of the vortex interaction with a stationary sphere [10] using the implicit fourth-order compact finite difference schemes for solving the flow with $R e=2000$. Paulo analyzed the vortex dynamics of the ring as it approached the sphere surface [10]. He identified that the boundary layer formed on the surface of the sphere undergoes separation to form a second vortex ring, which grow rapidly as it interact with the primary ring. However, most of the previous studies are restricted to a flat or a sphere. The vortex ring interaction with an ellipsoidal particle and particle's size effect on the evolution of the vortices have never been addressed in the literature.

In this work, we will investigate three-dimensional flow structure of a vortex ring impacting an ellipsoidal particle with different ellipticity (ratio between the major axis and the minor axis) and Reynolds numbers by using the lattice Boltzmann method (LBM) [11]. Lattice Boltzmann equation is able to recover the macroscopic Navier-Stokes equation, it looks like an artificial compressibility method (ACM) for solving the N-S equation [12]. However, in terms of spatial and temporal discretization, LBM is different from the ACM. For transient flows, the LBM may be more accurate for capturing the pressure waves than the ACM [12]. In the LBM, the Poisson equation is not required to solve. The LBM is an explicit scheme and the code is easy to be parallelized.

Here, through LBM simulations, we try to understand the mechanisms of the interaction between vortex ring and ellipsoidal particle. The effects of the ellipticity and $R e$ are investigated. 
This paper is organized as the following. Section 2 is a brief introduction of multiplerelaxation-time (MRT) lattice Boltzmann method, and corresponding boundary conditions. The vortex interaction with a flat wall was simulated to validate our numerical method through quantitative and qualitative comparison with experimental data. In Section 3, a vortex ring impacting an elliptical spheroid is studied. The effects of ellipticity and the $\operatorname{Re}(200 \leq \operatorname{Re} \leq 900)$ are investigated in detail and the evolution of the vortex structure is discussed. Finally, the conclusion is presented.

\section{Numerical method and the computational model}

In this section, our MRT-LBM will be validated through cases of a vortex ring impacting a flat wall. Macroscopically, the lattice Boltzmann equation is able to recover the incompressible N-S equation. The simplest lattice Boltzmann method is the Bhatnagar-GrossKrook (BGK) model, which is based on an approximation of a single relaxation time [13]. Until the 1990s, the MRT lattice Boltzmann method was developed, which overcomes some obvious defects of the BGK model, such as fixed ratio between the kinematic and bulk viscosities, improved the numerical stability [14].

The reasons why we use the MRT-LBM are illustrated in the follows. First, the LBM method is easy to implement and parallelized for this incompressible flow. Second, compared to conventional Navier-Stokes solvers of equal order of accuracy, the LBM has relatively low numerical dissipation and dispersion [12].

\subsection{MRT-LBM}

There are only two main steps in the code: streaming and collision. In the streaming step, the Distribution Functions (DF) in different directions (e.g., there are 19 components in the D3Q19 velocity model) at a computational node would propagate to its neighbourhood. The collision step is implemented locally. With the DF streaming from the neighbourhood, the macroscopic variables can be calculated as the moments of the DF. The DF can be updated through the collision step. In the collision step, the multiple-relax-time model [14] is used to ensure a better numerical stability.

In our study, the fluid flow is solved by the MRT-LBM [14]. The following MRT lattice Boltzmann (LB) equation [14] is employed to solve the incompressible NavierStokes equations,

$$
\left|f\left(\mathbf{x}+\mathbf{e}_{i} \Delta t, t+\Delta t\right)\right\rangle-|f(\mathbf{x}, t)\rangle=-M^{-1} \hat{\mathbf{S}}\left[|m(\mathbf{x}, \mathbf{t})\rangle-\left|m^{(e q)}(\mathbf{x}, \mathbf{t})\right\rangle\right],
$$

where the Dirac notation of ket $|\cdot\rangle$ vectors symbolize the column vectors. The particle distribution function $|f(\mathbf{x}, t)\rangle$ has 19 components $f_{i}$ with $i=0,1, \cdots, 18$ in our 3D simulations because the D3Q19 velocity model is used. $\mathbf{e}_{i}$ are the discrete velocities of the velocity model. The directions of the velocities are shown in Fig. 1(a), and for example, 
$\mathbf{e}_{1}$ can be written as $\mathbf{e}_{1}=(1,0,0) c$. Here $c=\Delta x / \Delta t$ is the lattice speed, where $\Delta x$ and $\Delta t$ are the lattice spacing $(1 l u)$ and time step $(1 t s)$ in LB simulations, respectively.

The collision matrix $\hat{\mathbf{S}}=M \cdot S \cdot M^{-1}$ is diagonal with $\hat{\mathbf{S}}$ [14] $\hat{\mathbf{S}} \equiv$ $\operatorname{diag}\left(0, s_{1}, s_{2}, 0, s_{4}, 0, s_{4}, 0, s_{4}, s_{9}, s_{10}, s_{9}, s_{10}, s_{13}, s_{13}, s_{13}, s_{16}, s_{16}, s_{16}\right) . \quad\left|m^{(e q)}\right\rangle$ is the equilibrium value of the moment $|m\rangle$. The $19 \times 19$ matrix $M$ is a linear transformation which is used to map a vector $|f\rangle$ in discrete velocity space to a vector $|m\rangle$ in moment space, i.e., $|m\rangle=M \cdot|f\rangle,|f\rangle=M^{-1} \cdot|m\rangle$. The matrix $M$, the discrete velocities of the D3Q19 model, and $\left|m^{(e q)}\right\rangle$ are all identical as those used by [14].

The macro-variables density $\rho$ and momentum $j_{\zeta}$ are obtained from

$$
\rho=\sum_{i} f_{i}, \quad j_{\zeta}=\sum_{i} f_{i} e_{i \zeta}
$$

where $\zeta$ denotes $x, y$, or $z$ coordinates. Here the collision process is executed in moment space [14]. In our simulations, the parameters are chosen as: $s_{1}=1.19, s_{2}=s_{10}=1.4, s_{4}=1.2$, $s_{9}=1 / \tau, s_{13}=s_{9}$, and $s_{16}=1.98$. The parameter $\tau$ is related to the kinematic viscosity of the fluid with $v_{f}=c_{s}^{2}(\tau-0.5) \Delta t$ and $c_{s}=c / \sqrt{3}$. The pressure in the flow field can be obtained from the density via the equation of state $p=c_{s}^{2} \rho$.

Macroscopically, (2.1) is able to recover the incompressible Navier-Stokes equation [14]. In the implementation to ensure the incompressibility condition, usually the velocity in the flow field should not exceed $0.1 c$.

\subsection{Boundary conditions}

As we know, the non-slip boundary condition should be ensured in the particle's surface. Here, the non-slip boundary condition in our study is based on the scheme of Lallemand [15].

In Fig. 1(b), the computational domain was separated by a solid surface. Some lattice nodes which inside the surface are solid nodes (filled black circles) and the collision steps are not implemented in these nodes. Outside the surface, there are fluid nodes, which are represented by the filled blue circles and black circles. The filled blue circle denote the fluid nodes which have at least one link of the 18 directions (shown in Fig. 1(a)) connecting with the solid nodes. Usually the half-way bounce back is used to ensure the non-slip boundary condition. In Fig. 1(b), we can see that after streaming step, there are 6 directions are unknown for the lattice node $\mathbf{b}$, i.e., $f_{1}(\mathbf{b}), f_{3}(\mathbf{b}), f_{5}(\mathbf{b}), f_{7}(\mathbf{b}), f_{15}(\mathbf{b})$, $f_{11}(\mathbf{b})$. For the half-way bounce back scheme (or simple bounce back), the unknown DF that comes from the solid node is set to be the DF in the reverse direction, which is already known. For example, $f_{1}(\mathbf{b})=f_{2}(\mathbf{b})$.

Here a more accurate curve wall boundary condition [15] is applied. In the follows, an example about how to get $f_{7}(\mathbf{b})$ is illustrated in detail and the other DFs can be obtained in the similar way. In Fig. 1(b), suppose the line ab intersects with the solid surface at point $\mathbf{p}$. $|\mathbf{b} \mathbf{p}|$ denotes the length of the green line $\mathbf{b} \mathbf{p}$. Here a parameter $q=|\mathbf{b} \mathbf{p}| /|\mathbf{a b}|$ is defined to describe the fraction in fluid region of a grid spacing intersected by the solid 

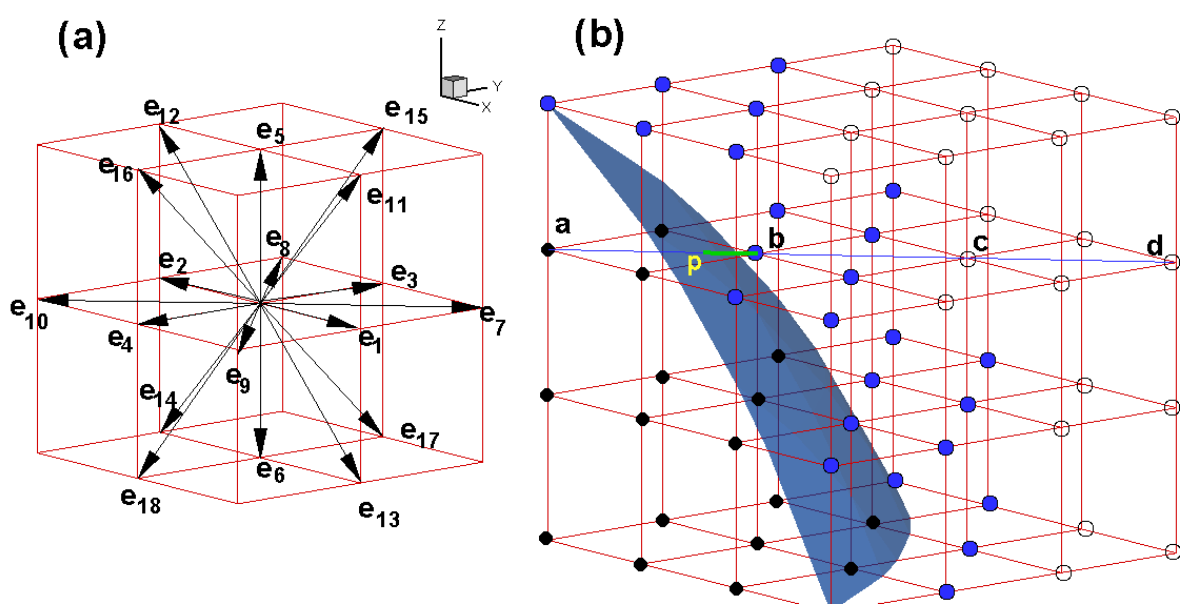

Figure 1: (Color online) (a) D3Q19 velocity model, $\mathbf{e}_{1}$ to $\mathbf{e}_{18}$ represent 18 non-zero components and $\mathbf{e}_{0}$ is a zero component and not labeled; (b) illustration of "interpolation bounce-back". The black filled circles are solid nodes, which are inside the surface of the solid body. The other circles are fluid nodes and the blue filled circles denote the fluid nodes which have at least one link with solid nodes (any of the 18 directions).

surface. After the streaming step, the unknown $f_{7}(\mathbf{b})$ can be obtained through a secondorder interpolation from the surrounding points [15]. For example, if $q<1 / 2, f_{7}(\mathbf{b})=$ $q(1+2 q) f_{10}(\mathbf{a})+\left(1-4 q^{2}\right) f_{10}(\mathbf{b})-q(1-2 q) f_{10}(\mathbf{c})$. More details about the "interpolation bounce back" can be found in [15].

\subsection{Computational model}

In this work, the size of the computational domain was $H \times H \times L$ in the $x, y$, and $z$ directions, respectively, where $H=12 r_{0}$ and $L=11 r_{0} . r_{0}$ is the initial radius of the ring and is used as a characteristic length. For an ellipsoidal particle $r_{1}, r_{2}, r_{3}$ denote the half of three major axes in the $x, y$, and $z$-directions, respectively. The equation of the ellipsoidal particle can be written as

$$
\frac{\left(x-x_{0}\right)^{2}}{r_{1}^{2}}+\frac{\left(y-y_{0}\right)^{2}}{r_{2}^{2}}+\frac{\left(z-z_{0}\right)^{2}}{r_{3}^{2}}=1
$$

where $\left(x_{0}, y_{0}, z_{0}\right)$ is the initial position of the ellipsoidal particle. As illustrated in Fig. 2, the vortex ring and the elliptical sphere are initially placed at a distance $z=9 r_{0}$ and $z_{0}=$ $7 r_{0}$, respectively. The spheroidal particle is placed exactly under the vortex ring center and the line connecting the centers of the vortex ring and the particle is on the $z$-axis.

The initial vorticity distribution of the vortex ring is assigned by a Gaussian function, i.e., the initial velocity field $\mathbf{u}_{0}=(u, v, w)_{0}$ is specified as [1]

$$
\mathbf{u}_{0}=\frac{\Gamma}{2 \pi \sigma}\left[1-e^{-\left(\sigma / \sigma_{0}\right)^{2}}\right] \boldsymbol{\theta}
$$




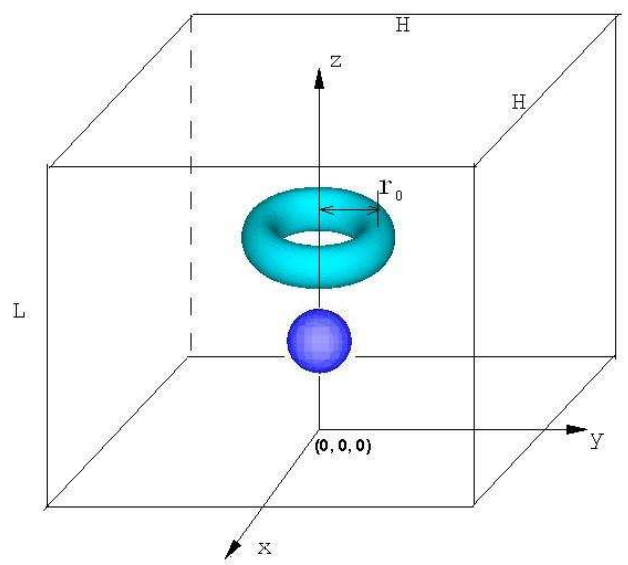

Figure 2: Computation model for a vortex ring approaching a spheroidal particle. $r_{0}$ is the initial radius of the vortex ring.

where $\Gamma$ is the circulation of the ring, $\theta$ is the unit vector tangential to circulation circles of the vortex ring core, $\sigma$ is the radial distance from the center of the core and the initial one is $\sigma_{0}=\alpha r_{0}$. The corresponding initial translational speed of the vortex ring $w_{c}$ is approximately [16]

$$
w_{c}=\frac{\Gamma}{4 \pi r_{0}}\left(\ln \frac{8 r_{0}}{\sigma_{0}}-\frac{1}{4}\right) .
$$

The non-dimensional variables, the Reynolds number of the vortex ring, the position $\mathbf{R}=(X, Y, Z)$, the vorticity $\Omega$, time $T$, and pressure $P$ are defined as

$$
R e \equiv \frac{2 u_{c} r_{0}}{v}, \quad \mathbf{R} \equiv \frac{\mathbf{r}}{r_{0}}, \quad \mathbf{\Omega} \equiv \frac{\boldsymbol{\omega} r_{0}^{2}}{\Gamma_{0}}, \quad T \equiv \frac{\Gamma_{0} t}{r_{0}^{2}}, \quad P \equiv \frac{r_{0}^{2}\left(p-p_{\infty}\right)}{\rho \Gamma_{0}^{2}},
$$

where $\mathbf{r}=(x, y, z)$ is the spatial vector in the coordinates system, $\boldsymbol{\omega}=\left(\omega_{x}, \omega_{y}, \omega_{z}\right)$ is the vorticity.

In all of our simulations, constant-pressure boundary condition is applied on the six faces of the computational domain. For the boundary condition, the density of the fluid is specified as unity and the velocity is extrapolated from the inner fluid nodes. Then the equilibrium distribution function in the boundary fluid node can be calculated. The non-equilibrium part of the distribution function is extrapolated from the inner fluid nodes [17].

\subsection{Validation of the numerical method}

To validate our code, grid-independence study was performed first. The vortex ring impacting a flat wall is simulated. As we know, some previous studies $[1,3,8]$ demonstrate that for intermediate $\operatorname{Re}(\operatorname{Re}<1000)$, as long as $H$ and $L \geq 10 r_{0}$, the effect due to finite domain is negligible. Here, $H=12 r_{0}$ and $L=11 r_{0}$ are adopted. In the grid-independence 
Table 1: Peak value of the vorticity in the $(y, z)$-plane at dimensionless time $T=10,12,14$ for different meshes.

\begin{tabular}{||c|c|c|c|c||}
\hline & $r_{0}=20 l u$ & $r_{0}=25 l u$ & $r_{0}=30 l u$ & $r_{0}=35 l u$ \\
\hline$T=10$ & 4.53626 & 4.67464 & 4.71982 & 4.73279 \\
\hline$T=14$ & 4.60296 & 4.75269 & 4.75864 & 4.76046 \\
\hline$T=18$ & 5.18556 & 5.24038 & 5.25373 & 5.25698 \\
\hline
\end{tabular}

study, four different meshes are used. The mesh resolutions are $r_{0}=20 l u, 25 l u, 30 l u$, and $35 \mathrm{lu}$, respectively. The corresponding domain sizes in the simulations are $240 \times 240 \times 220$, $300 \times 300 \times 275,360 \times 360 \times 330,420 \times 420 \times 385$ respectively. In the simulations, the vortex ring is initially put $3 r_{0}$ above the flat wall, $\alpha=0.21$ and $R e=830$.

The peak values of vorticities in the $(y, z)$-plane at different times are compared and shown in Table 1. From Table 1, we can see that at the times listed, there are significant discrepancies between the case of $r_{0}=20 l u$ and other cases (with maximum $3.2 \%$ discrepancy). On the other hand, the discrepancies between cases of $r_{0}=30 \mathrm{lu}$ and $r_{0}=35 \mathrm{lu}$ are much smaller (less than $0.3 \%$ ) and negligible. The result shows that the grid with resolution $r_{0}=30 l u$ is fine enough to carry out relevant numerical study. Hence, in the following study, the mesh resolution is set to be $r_{0}=30 l u$, i.e., the domain size is $360 \times 360 \times 330$.

To further validate our code, the trajectory of the primary vortex ring core is compared with the experimental data for the case $R e=830$. The location of the primary vortex $(\bar{y}, \bar{z})$ is calculated through

$$
\bar{y}=\frac{1}{\Gamma} \int_{A} y \omega_{x} d y d z, \quad \bar{z}=\frac{1}{\Gamma} \int_{A} z \omega_{x} d y d z,
$$

where $A \in[0, H / 2] \times L$ in the $(y, z)$-plane. As shown in Fig. 3, the LBM trajectory $\left(Y=\bar{y} / r_{0}\right.$, $\left.Z=\bar{z} / r_{0}\right)$ fits very well with the experiment data [5].

The evolution of the vortex is also compared with the experimental data and shown in Fig. 4. We can see the when the primary vortex ring approaches the flat wall, the secondary vortex is generated from the wall and rolls up ((a) and (b) in the figure). With the

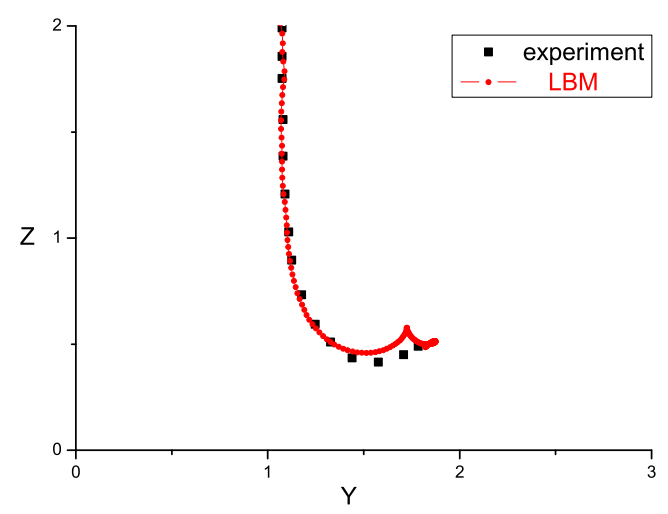

Figure 3: The trajectory of the primary votex ring center for the case $R e=830$. The line and squares denote our LBM result and the experimental data [5], respectively. $Y$ and $Z$ are normalized by $r_{0}$. 


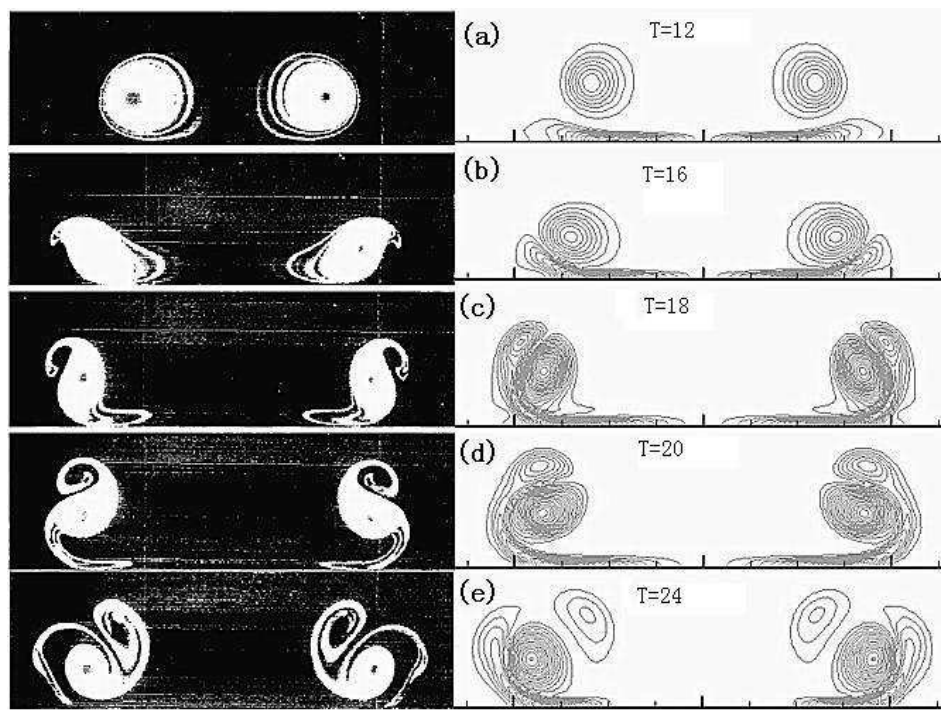

Figure 4: The evolution of the vortex impacting a flat for $R e=830$. The left and right columns are the experimental data [5] and our LBM result, respectively.

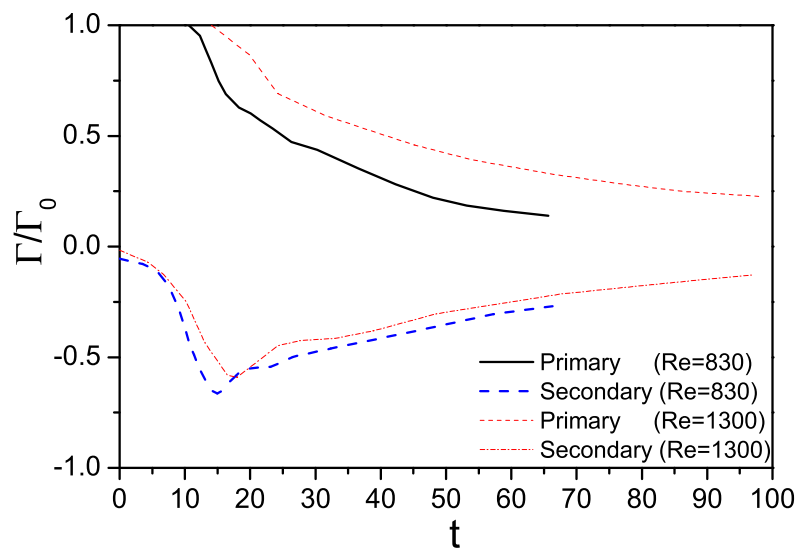

Figure 5: The circulation as a function of time in the $(y, z)$-plane. The thick solid and dash lines represent the circulation of the primary and secondary vortices of $R e=830$, respectively. The thin dash and dashdot lines are those of $\operatorname{Re}=1300 . \Gamma_{0}$ is the initial circulation of the primary vortex ring. The result of $\operatorname{Re}=1300$ comes from [3].

expansion of the primary vortex, the secondary vortex rolls up to the top of the primary vortex ((c) and (d)). Even the tertiary vortex structures can be seen in the last row of the figure.

Finally, the circulation of the primary and the secondary vortices in the $(y, z)$ symmetry plane for the case $R e=830$ is compared with the data of Orlandi and Verzicco [3]. Although the data is from the case of $R e=1300$, the circulation as a function of time for the $R e=830$ is expected to be similar to that of $R e=1300$. From Fig. 5, we can see that our $\mathrm{LBM}$ result for $R e=830$ is similar to the data for $R e=1300$. 


\section{Results and discussion}

In this section, first a vortex ring impacting a sphere is studied. Effect of sphere size (the size of vortex ring is fixed) on the evolution of vortex structure is discussed. Then, the geometric effect due to the elliptical spheroid is investigated. The Re-effect is also addressed. The dynamics of the flow, e.g., the evolution of the vortices, are analyzed. In the following study, $\alpha$ is set to be 0.45 , which means the primary vortex ring is thick.

\subsection{Effect of sphere size}

In this section, the vortex ring impacting a sphere with different sphere size is studied. Firstly, $r_{1}=r_{2}=r_{3}=4 / 15 r_{0}$ is investigated. Fig. 6 shows the evolution of the vortex ring for $R e=600$.

As shown in Fig. 6(1), when the vortex ring approaches the sphere, the intersection of the ring core is deformed to be an elliptical shape. Then, a very thin boundary layer is generated near the sphere (Fig. 6(2)). In Fig. 6(3), the boundary layer stretches and it rapidly grows and separates from the spheroid. At the same time, the radius of the primary ring increases in response to the presence of sphere and the production of adverse pressure gradient at the solid wall [1]. As the interaction continues, the boundary
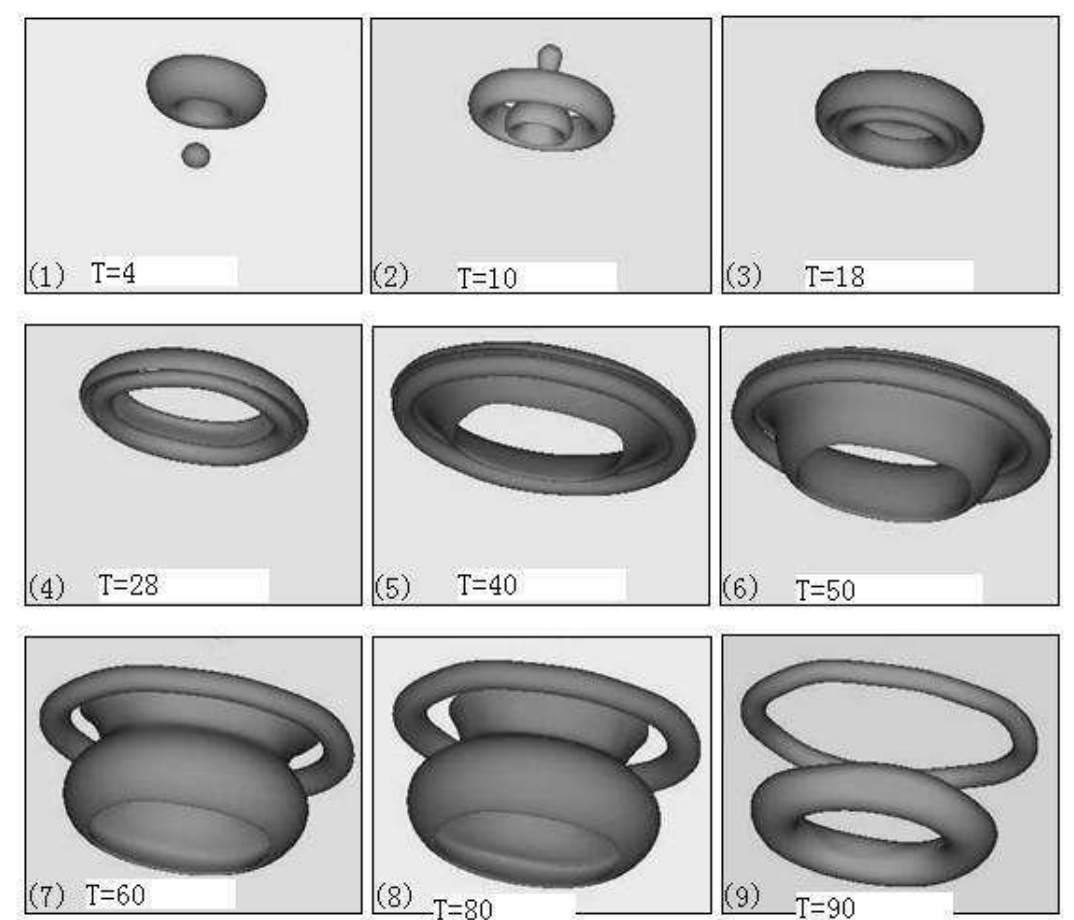

Figure 6: Vortex structure evolution of the ring impacting a sphere for $R e=600$ and the radius of the sphere is $4 r_{0} / 15$. 


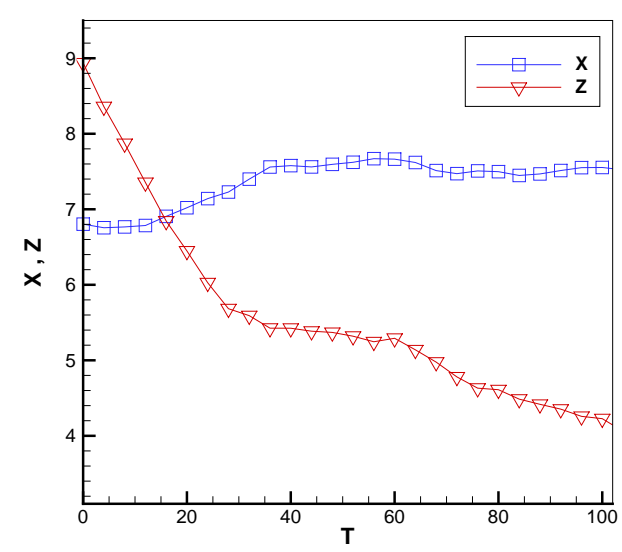

Figure 7: Primary vortex ring location $x$ and $z$ as a function of time in the $(x, z)$-plane.

layer rolls up from the sphere and forms a secondary vortex. Since these two vortex rings have opposite sign vorticity, the secondary vortex expands rapidly and the primary and secondary vortex rings wrap around each other. Figs. 6(4) and (5) show that the primary ring starts to decelerate considerably and the secondary-vortex ring revolves as a satellite around the outside of the primary vortex core. Due to the secondary vortex, the primary ring was elongated and lost its elliptical form of the ring core and its radius does not increase as shown in Fig. 6(6). At this point, the primary ring stops its forward moving (the $-z$-direction), while the remainder of the secondary vortex ring retains its link with the solid surface and may evolve a tertiary vortex but it is too weak to visualize [1]. As shown in Figs. 6(7) and (8), the elongated primary ring forms a "wheel hub" structure and pinches off. The ring core changes to an elliptical shape again. Then, the primary ring restarts its forward motion and the radius reaches an approximately constant value. Finally, two distinct vortex rings are formed in Fig. 6(9). The primary ring continues its forward movement at a fixed velocity and the second ring is almost stationary around the sphere.

We explain why the second ring is almost stationary in the following. Obviously, the circulations of the secondary and the primary vortices are opposite, and the induced translational velocities are also opposite. On the other hand, the secondary vortex is weak and inside the velocity field dominated by the primary vortex. The induced upward translational velocity of the secondary vortex may be canceled by the downward velocity induced by the primary vortex. Hence, the secondary vortex may be stationary at that time interval we are interested in.

The evolution of the radius and translational velocity of the ring can be analyzed in detail in the follows. Fig. 7 shows the locations of $x$ and $z$ of the primary vortex ring, which were obtained by tracking the peak vorticity of the vortex center in the $(x, z)$-plane. It is seen that when the ring approaches the sphere, the velocity of the ring keeps constant and the displacement of $x$ stays zero. As the ring goes closer to sphere, its radius begins 


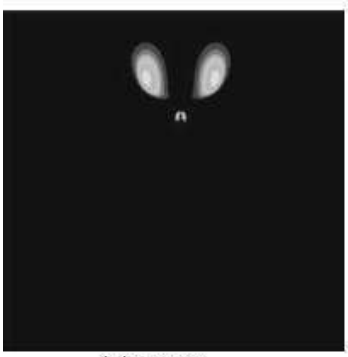

(a) $\mathrm{T}=10$

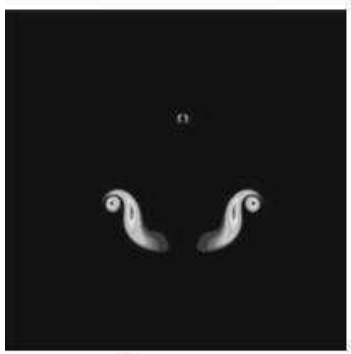

(d) $\mathrm{T}=60$

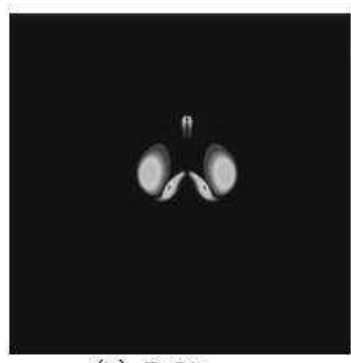

(b) $\mathrm{T}=20$

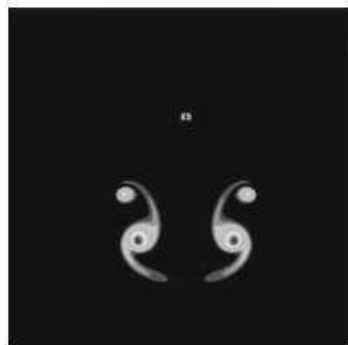

(e) $\mathrm{T}=90$

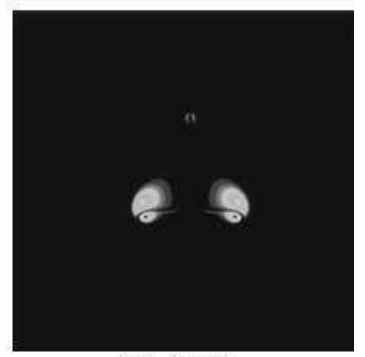

(c) $\mathrm{T}=40$

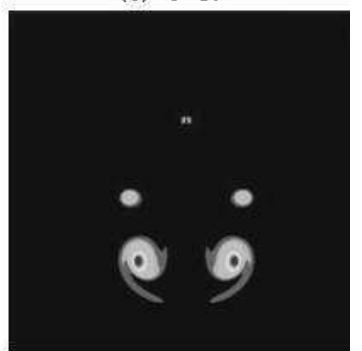

(f) $\mathrm{T}=120$

Figure 8: The evolution of vortex impacting a small sphere in the $(x, z)$-plane.

to expand. During this time, the boundary layer starts to roll up and gradually lead to the secondary vorticity. At $T=35$, the primary ring nearly stops its forward motion. In this moment, the primary and secondary vortex rings wrap around each other. About the time of $T=60$, the primary ring pinches off. It restarts its forward motion with an elliptical ring core. The translational velocity is smaller compared to the initial speed. Finally, the ring moves with a constant velocity and its radius almost reaches a constant.

Cases of spheres with smaller radius $r_{1}=r_{2}=r_{3}=r_{0} / 6$ and $r_{0} / 15$ are simulated. Fig. 8 shows the evolution of vortex rings with $r_{1}=r_{2}=r_{3}=r_{0} / 15$ in the $(x, z)$-plane, which is qualitative similar to the behavior of the vortex rings in the case of $r_{1}=r_{2}=r_{3}=4 r_{0} / 15$, except for the magnitude of vorticity.

Fig. 9 shows a quantitative comparison of the trajectories of the cases $r_{1}=r_{2}=r_{3}=r_{0} / 6$ (open symbols) and $r_{1}=r_{2}=r_{3}=4 r_{0} / 15$ (filled symbols). For the case of $r_{0} / 6$, the generated secondary vortex detaches from the sphere later than the case of $4 r_{0} / 15$. It seems the radius of the sphere would affect the final position of the secondary vortex. For a smaller sphere, the secondary vortex stays far away from the sphere. That is because when the sphere is large, the secondary vortex which is generated from the sphere experiences a stronger vortex stretching.

\subsection{Effect of the ellipticity of the ellipsoidal particle}

The effect of ellipticity (ratio of long axis to short axis) of the particle is studied in this section. Here, the $R e=600$ is fixed. The evolution of vortex rings for the case of ellipsoidal particle $r_{1}=r_{3}=r_{0} / 6, r_{2}=7 r_{0} / 30$ is shown in Fig. 10. The evolution pattern is similar to 


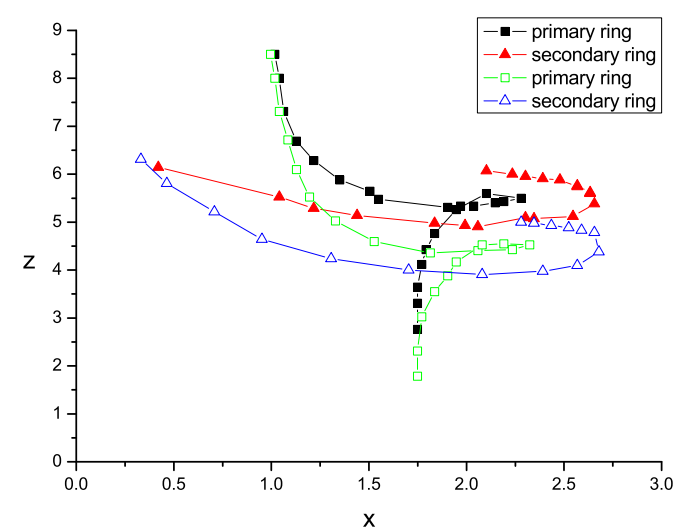

Figure 9: The trajectory of the vortex rings impacting different spheres. Filled symbols represent the case of $r_{1}=r_{2}=r_{3}=4 r_{0} / 15$ and open symbols represent the case of $r_{1}=r_{2}=r_{3}=r_{0} / 6$.

that in the case of a sphere. When the ring approaches the particle, it induces a boundary layer at the wall, which results in a secondary vortex, as shown in Fig. 10(3). In Figs. 10(4) and (5), the primary vortex ring will expand, but the expansion along the long axis is stronger than that in the short axis. The primary and secondary vortices begin to wrap around each other and continuously expand. Subsequently, the primary ring becomes non-uniform along the circumference direction and the expansion along the two axes $(x$ - and $y$-axes) is different. Then the primary vortex ring wraps around the secondary vortex, leading to a vortex structure like a flower (Fig. 10(6)). Later, the "flower" begins to shrink and pass through the secondary vortex, as shown in Fig. 10(7). Hence, in the flow field, two distinct vortex rings appear. The primary vortex ring resumes to translate along $z$-direction and the secondary vortex ring is almost stationary. Finally, both the primary and secondary vortex rings all deformed into elliptical shapes. They are stretched along the short and long axes of the spheroid, respectively, as shown in Fig. 10(8).

To quantitatively study the effect of particle's shape, cases $r_{1}=r_{3}=r_{0} / 6, r_{2}=3 r_{0} / 10$ were also simulated. Figs. 11(a) and (b) show the evolution of the vortex ring in the $(x, z)$ and and $(y, z)$ symmetric planes, respectively. From these slices, it is seen the secondary vortex ring mainly stretches along the long axis of the particle. In the column (3) of the figure, the primary vortex also stretches along the long axis. However, from column (4) and (5), we can see that after the primary vortex ring is recovered, the primary vortex is mainly stretching in the $(x, z)$-plane, i.e., along the $x$-axis, which is the short axis of the particle. The possible reason is that the secondary vortex stretching along the long axis of the particle delays the recovery of the primary vortex in the direction. Hence, finally when the primary and secondary vortices become elliptical, their main axes are perpendicular.

The case of a smaller ellipsoidal particle $r_{1}=r_{3}=r_{0} / 6, r_{2}=r_{0} / 10$ is simulated. The trajectories of the vortex rings at different $r_{1} / r_{2}$ are shown in Fig. 12. It is found the vortex evolution is close to that of a sphere (Fig. 12(b)). Due to the smaller size of the particle, the 

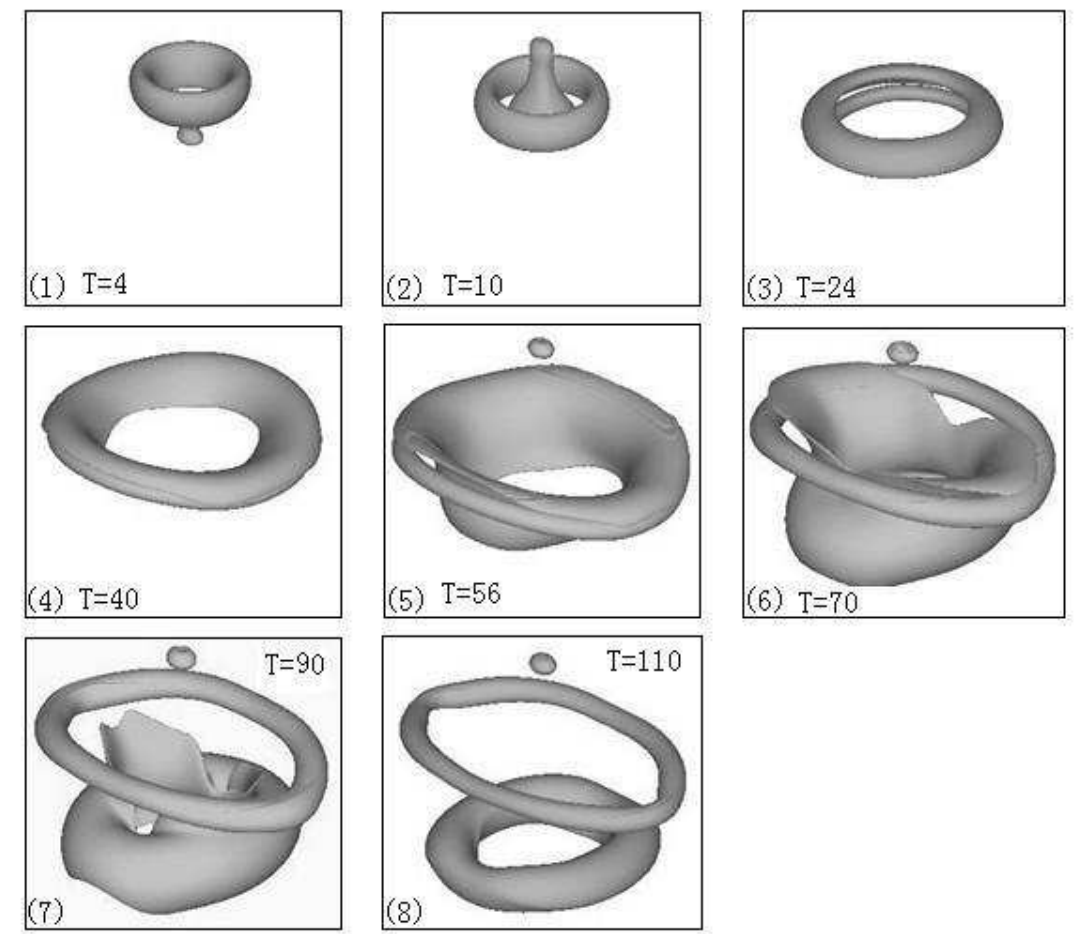

Figure 10: The evolution of a ring impacting an elliptical spheroid for $R e=600$.

(a)<smiles>[Li][Hg]</smiles>

(b)<smiles></smiles>

(1)

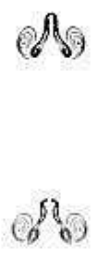

(2)

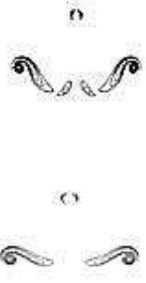

(3)

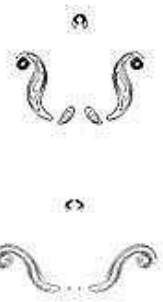

(4)

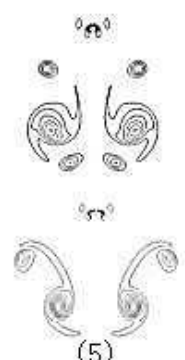

(5)

Figure 11: Vortex contours for an ellipsoidal particle with $r_{1}=r_{3}=r_{0} / 6, r_{2}=3 r_{0} / 10$. (a) the $(x, z)$-plane; (b) the $(y, z)$-plane.

different lengthes of the ellipsoid in the $x$ and $y$ axes seem not affect the behavior of the vortex much compared with the cases of a sphere. From Fig. 12(a), we can see that when the particle is large and the ellipticity $r_{1} / r_{2}$ is far from unity (sphere cases), the primary and secondary rings become more elliptical in the $(x, y)$-plane.

\subsection{Effect of $R e$}

In this part, we study the evolution of the flow on different $R e$ with a fixed radius $r_{1}=r_{3}=$ $r_{0} / 6, r_{2}=7 r_{0} / 30$. For the case of the $R e=200$, Fig. 13 shows the flow does not generate a 

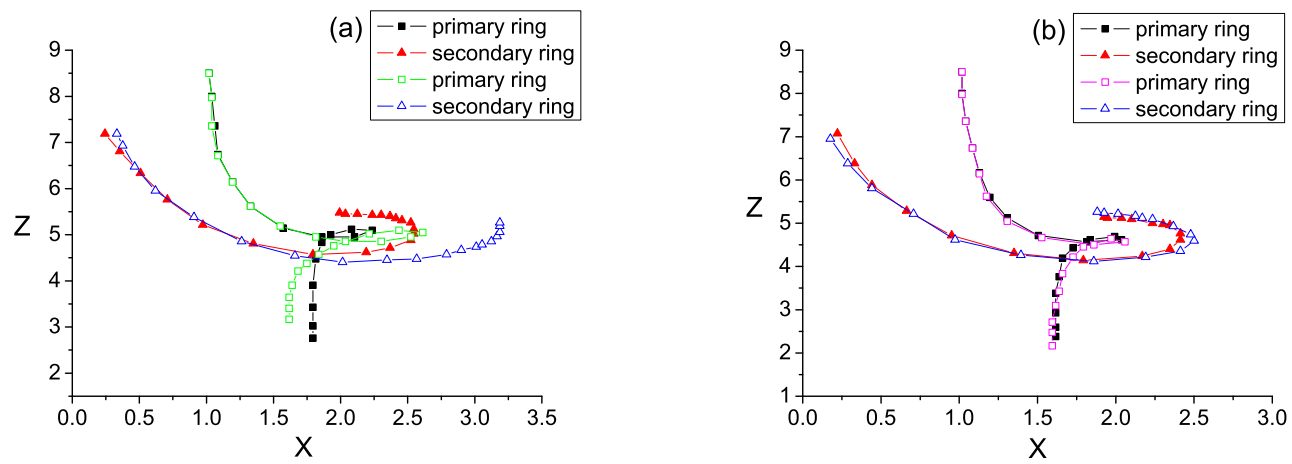

Figure 12: The trajectory of the vortex rings at different radical ratio. Filled symbol represent the trajectory in the $(x, z)$-plane and open symbol represent those in the $(y, z)$-plane. (a) $r_{2}=3 r_{0} / 10=9 l u$; (b) $r_{2}=r_{0} / 10=3 l u$. In both cases, $r_{1}=r_{3}=r_{0} / 6$.

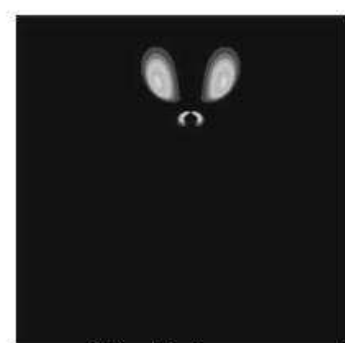

(a) $\mathrm{T}=8$

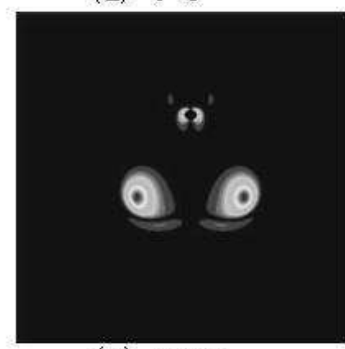

(d) $\mathrm{T}=50$

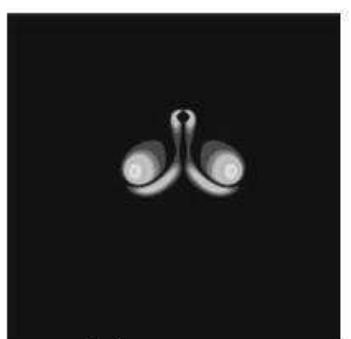

(b) $\mathrm{T}=20$

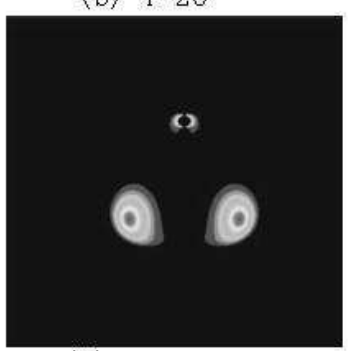

(e) $\mathrm{T}=70$

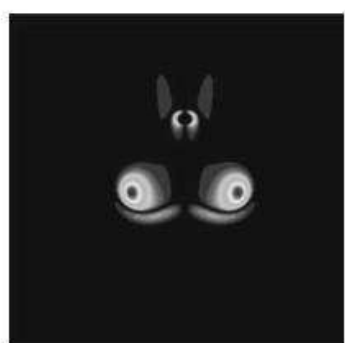

(c) $\mathrm{T}=34$

Figure 13: The evolution of a vortex ring impacting an elliptical sphere with $R e=200$ in the $(y, z)$-plane.

secondary vortex because the viscous effect is so strong that it diffuses the vorticity and weakens the intensity of the ring. In other words, the induced vorticity in the boundary of the solid is quickly dissipated. Hence, only the primary ring moving forward in the evolution.

For the case of $R e=400$. As the viscous dissipation becomes weaker, the vortex-solid interaction shows the same phenomenon as illuminated in Fig. $10(R e=600)$. When $R e=900$, a more complicated phenomenon appears. Due to the high $R e$, the inertial of the flow is more prominent and viscous dissipation is relatively small. Before the ring approaches the solid, its circulation holds the primary value. When the ring impacts the particle, the secondary vortex is detached from the wall faster than cases of small Re. It 


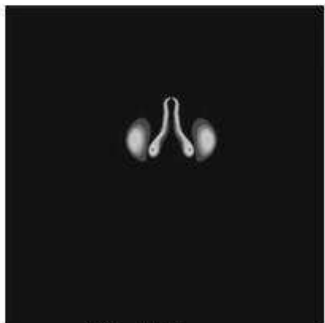

(a) $\quad T=8$

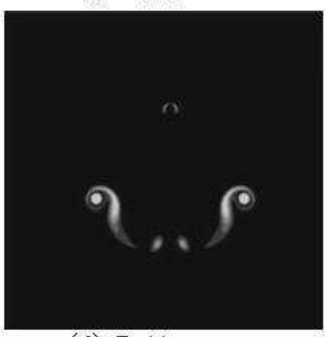

(d) $\mathrm{T}=44$

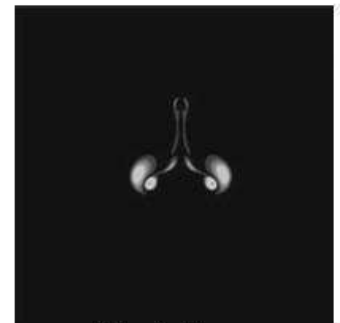

(b) $\mathrm{T}=12$

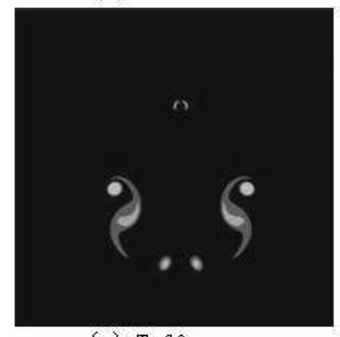

(e) $\mathrm{T}=60$

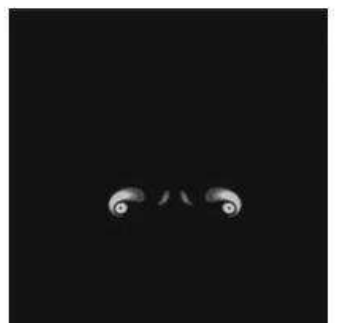

(c) $\mathrm{T}=20$

Figure 14: The evolution of a vortex ring impacting an elliptical sphere with $R e=900$ in the $(y, z)$-plane.
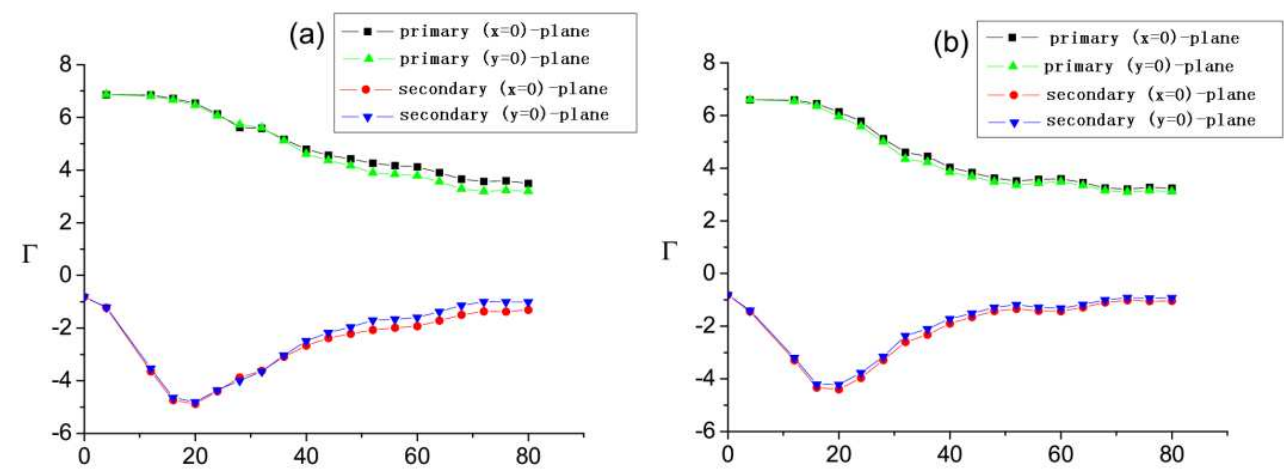

Figure 15: Circulation of the primary and secondary vortex in $(y=0)$-plane $(x$-axis $)$ and $(x=0)$-plane ( $y$-axis $)$ as a function of time. (a) $R e=900$, (b) $R e=400$. In both cases, $r_{1}=r_{3}=r_{0} / 6, r_{2}=7 r_{0} / 30$.

is stronger and moves faster. As we can see in Fig. 14, except the primary and secondary vortices, a tertiary vortex ring is also separated from the boundary layer.

The relative circulations in the $(x=0)$ - and $(y=0)$-planes for cases of $R e=900$ and $R e=400$ are shown in Fig. 15. For the case of $R e=900$, initially the circulation of the primary vortex in the $(x=0)$ - and $(y=0)$-planes are identical. After the secondary vortex generated, as shown in Fig. 15(a), the circulation values of the primary and secondary vortices in the $(x=0)$-plane are larger than those in the $(y=0)$-plane. For $R e=400$, Fig. 15(b) demonstrates that the circulation values of the primary and secondary vortices in the $(x=0)$-plane are almost identical to those in the $(y=0)$-plane. Hence, the primary and secondary vortex rings are stretched more strongly in the former case $(R e=900)$. 


\section{Conclusions}

In this paper, three-dimensional vortex ring impacting an ellipsoidal particle is studied using the MRT-LBM. The evolution of the vorticity on the symmetric planes, trajectories of vortex center, the effects of particle size, $R e$ and the ellipticity on the evolution of vortex structure are discussed in detail.

When the vortex ring impacting with a spheroidal particle, the interaction is described as the following three stages. Firstly, the secondary vortex is generated as the stretching of boundary vorticity at the beginning of impacting. In the second stage, the primary and secondary vortices expand and shrink radically and will wrap around each other. Finally, the primary vortex ring will recover from the 'wheel hub' and 'flower' structure for a sphere and a prolate particle, respectively.

The trajectories of the primary and secondary vortices demonstrate that the wrapping pattern of primary and secondary vortex rings is very different from the situation of the vortex ring impacting a plate. The evolution of vortex structure seems sensitive to the size of the particle. When the particle is smaller, the primary ring expands slowly and eventually, the secondary vortex stays further from the sphere.

When the vortex ring impacting with a prolate spheroid, in the vortex wrapping stage, the primary vortex recovers along the short axis of the particle faster than the long axis direction. The possible reason is that the secondary vortex stretching along the long axis of the particle delays the recovery of the primary vortex in the direction. Eventually, the primary vortex mainly expands along the short axis of the particle.

For cases with larger ellipticity, it is more significant that the primary and secondary vortices are mainly stretching along the short and long axes of the particle, respectively. The Re also plays an important role in the vortex evolution. The second vortex is very weak at small-Re cases while a more complicated vorticity structure involving a tertiary vortex ring is generated at large- $R e$ cases.

In the future, our study will be extended to vortex interaction with a moving particle $[9,18]$.

\section{Acknowledgments}

This work was supported by the National Science Foundation of China (NSFC, No. 11172297).

\section{References}

[1] M. ChenG, J. LOU AND L. S. LUO, Numerical study of a vortex ring impacting a flat wall, J. Fluid Mech., 660 (2010), pp. 430-455.

[2] T. T. Lim, T. B. NicKELS AND M. S. CHONG, A note on the cause of rebound in the head-on collision of a vortex ring with a wall, Exp. Fluids, 12 (1991), pp. 41-48. 
[3] P. ORlandi AND R. VerzicCO, Vortex rings impinging on walls: axisymmetric and threedimensional simulations, J. Fluid Mech., 256 (1993), pp. 615-646.

[4] A. M. NAguib AND M. M. KoOchesfahani, On wall-pressure sources associated with the unsteady separation in a vortex-ring/wall interaction, Phys. Fluids, 16 (2004), pp. 2613-2622.

[5] C. C. CHU, C. T. WANG AND C. C. CHANG, A vortex ring impinging on a solid plane surfaceVortex structure and surface force, Phys. Fluids A, 7 (1995), pp. 1391-1401.

[6] P. G. SAFFMAN, The approach of a vortex pair to a plane surface in inviscid fluid, J. Fluid Mech., 92 (1979), pp. 497-503.

[7] K. KiYA, M. OHYAMA AND J. C. R. HUNT, Vortex pairs and rings interacting with shear-layer vortices, J. Fluid Mech., 172 (1986), pp. 1-15.

[8] C. H. LiU, Vortex simulation of unsteady shear flow induced by a vortex ring, Comput. Fluids, 31 (2002), pp. 183-207.

[9] J. J. ALLEN, Y. JOUANNE AND B. N. SHASHIKANTH, Vortex interaction with a moving sphere, J. Fluid Mech., 587 (2007), pp. 337-346.

[10] P. J. S. A. FERREIRA DE SOUSA, Three-dimensional instability on the interaction between a vortex and a stationary sphere, Theor. Comput. Fluid Dyn., (2011), 11-0239-5.

[11] D. YU, R. MEI, L. S. LuO AND W. SHYY, Viscous flow computations with the method of lattice Boltzmann equation, Prog. Aerosp. Sci., 39 (2003), pp. 329-367.

[12] X. He AND G. D. Doolen ET AL., Comparison of the lattice Boltzmann method and the artificial compressibility method for Navier-Stokes equations, J. Comput. Phys., 179 (2002), pp. 439-451.

[13] P. L. Bhatnagar, E. P. GROSS AND M. KROOKM, A model for collision processes in gases 1, Small amplitude processes in charged and neutral one-component systems, Phys. Rev., 94 (1954), pp. 511-525.

[14] D. D'Humieres, I. GinzburG, M. KrafczyK, P. Lallemand, AND L-S Luo, Multiplerelaxation-time lattice Boltzmann models in three dimensions, Phil. Trans. Royal Society of London: Series A, 360 (2002), pp. 437-451.

[15] P. LAllemand AND L-S LuO, Lattice Boltzmann method for moving boundaries, J. Comput. Phys., 184 (2003), pp. 406-421.

[16] H. LAMB, Hydrodynamics, Cambridge University Press.

[17] H. B. HuANG, X. YANG, M. KRAFCZYK AND X. Y. LU, Rotation of spheroidal particles in Couette flows, J. Fluid Mech., 692 (2012), pp. 369-394.

[18] Y. CHen, Q. D. CAI, Z. H. XIA, M. R. WANG AND S. Y. CHEN, Momentum-exchange method in lattice Boltzmann simulations of particle-fluid interactions, Phys. Rev. E, 88 (2013), 013303. 